\title{
Correction: LotuS: an efficient and user-friendly OTU processing pipeline
}

Falk Hildebrand ${ }^{1,2,3}$, Raul Y Tito ${ }^{1,2,6,7}$, Anita Yvonne Voigt ${ }^{3,4}$, Peer Bork ${ }^{3,5}$ and Jeroen Raes ${ }^{1,2,6,7^{*}}$

\section{Correction}

The name of the second author was incorrect in the original published article. The correct author name is Raul Y Tito.

\begin{abstract}
Author details
'Department of Structural Biology, Vlaams Instituut voor Biotechnologie (VIB), Pleinlaan 2, Brussels 1050, Belgium. ${ }^{2}$ Department of Bioscience Engineering, Vrije Universiteit Brussel, Pleinlaan 2, Brussels 1050, Belgium. ${ }^{3}$ Structural \&

Computational Biology Unit, European Molecular Biology Laboratory (EMBL), Meyerhofstrasse 1, Heidelberg 69117, Germany. ${ }^{4}$ Molecular Medicine

Partnership Unit (MMPU), University of Heidelberg and European Molecular Biology Laboratory, Heidelberg, Germany. ${ }^{5}$ Max Delbrück Centre for Molecular Medicine, Robert-Rössle-Str. 10, Berlin 13125, Germany. ${ }^{6}$ Department of Microbiology and Immunology, REGA institute, KU Leuven, Herestraat 49, Leuven 3000, Belgium. ' VIB Center for the Biology of Disease, Herestraat 49, Leuven 3000, Belgium.
\end{abstract}

Received: 9 October 2014 Accepted: 9 October 2014

Published: 14 October 2014

\section{Reference}

1. Hildebrand F, Tito TY, Voigt AY, Bork P, Raes J: Lotu S: an efficient and user-friendly OTU processing pipeline. Microbiome 2014, 2:30.

doi:10.1186/2049-2618-2-37

Cite this article as: Hildebrand et al:: Correction: LotuS: an efficient and user-friendly OTU processing pipeline. Microbiome 2014 2:37.

* Correspondence: jeroen.raes@med.kuleuven.be

'Department of Structural Biology, Vlaams Instituut voor Biotechnologie (VIB), Pleinlaan 2, Brussels 1050, Belgium

${ }^{2}$ Department of Bioscience Engineering, Vrije Universiteit Brussel, Pleinlaan 2, Brussels 1050, Belgium
Submit your next manuscript to BioMed Central and take full advantage of:

- Convenient online submission

- Thorough peer review

- No space constraints or color figure charges

- Immediate publication on acceptance

- Inclusion in PubMed, CAS, Scopus and Google Scholar

- Research which is freely available for redistribution

Submit your manuscript at www.biomedcentral.com/submit 